\title{
1 Dual stochasticity in the cortex as a biologically plausible learning with the most
}

\section{2 efficient coding}

4 Jun-nosuke Teramae

5 Graduate School of Informatics, Kyoto University, Kyoto, Japan

6 teramae@acs.i.kyoto-u.ac.jp

\section{Abstract}

9 Neurons and synapses in the cerebral cortex behave stochastically. The advantages of 10 such stochastic properties have been proposed in several works, but the relationship and 11 synergy between the stochasticities of neurons and synapses remain largely unexplored.

12 Here, we show that these stochastic features can be inseparably integrated into a simple 13 framework that provides a practical and biologically plausible learning algorithm that 14 consistently accounts for various experimental results, including the most efficient 15 power-law coding of the cortex. The derived algorithm overcomes many of the 16 limitations of conventional learning algorithms of neural networks. As an experimentally 17 testable prediction, we derived the slow retrograde modulation of the excitability of 18 neurons from this algorithm. Because of the simplicity and flexibility of this algorithm, 19 we anticipate that it will be useful in the development of neuromorphic devices and 20 scalable AI chips, and that it will help bridge the gap between neuroscience and machine 21 learning.

\section{Introduction}

24 Neurons in the cortex continuously generate irregular spike trains with fluctuating 25 membrane potentials and greatly varying firing rates, even across trials in which an 26 animal exhibits appropriate responses and learning in a precisely repeatable manner [1-7].

27 It has also been found that synapses in the cerebral cortex behave stochastically [8]. The 28 formation, elimination and volume change of dendritic spines exhibit random 29 fluctuations [9-15]. The release of neurotransmitter from synapses is also an inherently 30 stochastic process [16-18].

32 Theoretically, it has been pointed out that algorithms incorporating these stochastic 33 features can carry out nearly optimal computation in a noisy environment through 
34 Bayesian inference [19-28]. To this time, however, the stochastic behaviors of neurons

35 and synapses have been studied separately. It remains unclear if the apparent advantage

36 gained from stochasticity depends on both neurons and synapses behaving stochastically,

37 and if so, whether there is a synergetic interaction between these two types of stochastic

38 behavior. It is also uncertain how learning generated by stochastically functioning

39 neurons and synapses can yield appropriate and precisely repeatable behavioral

40 responses.

42 In this paper, we show that the stochastic behaviors of neurons and synapses can be

43 inseparably integrated into a simple framework of a sampling-based Bayesian inference

44 model, in which their synergy provides an effective and flexible learning algorithm that is

45 consistent with various experimental findings of the cortex. The derived algorithm

46 accurately describes the plasticity of cortical synapses [29, 30], while it faithfully

47 generates the extremely different timescales of neural and synaptic dynamics, the

48 higher-order statistics of the topology of local cortical circuits [31], and the response

49 properties of cortical neurons, including Gabor-filter-like receptive fields [32, 33], a

50 positive relationship between the receptive field correlation and average connection

51 weight between neurons [34], and the nearly optimal power-law scaling of population

52 activity of neuron [7]. These results strongly suggest that the stochastic behaviors of

53 neurons and synapses are both essential attributes of neural computation and learning. As

54 an experimentally testable prediction of the proposed model, we derive the slow

55 retrograde modulation of the excitability of neurons by postsynaptic neurons. As far as

56 the author is aware, this is the first prediction of its kind and experiments to verify the

57 existence of such slow retrograde modulation have not yet been attempted.

59 The proposed algorithm can be regarded as a natural integration of the conventional

60 learning theories, error backpropagation learning [35, 36], Bayesian inference [21],

61 Boltzmann machine learning [37], and reinforcement learning [38]. This algorithm can

62 be regarded as an extension of a Boltzmann machine, and it acts as a stochastic variant of

63 error backpropagation. However, the proposed algorithm overcomes most of the

64 limitations that caused backpropagation not to be regarded as the learning principle of the

65 brain. Unlike backpropagation learning, the proposed algorithm does not require neither

66 objective functions, the fine-tuning of parameters, coordinated or synchronous updates of 
67 variables, a feed-forward network structure, or the alternating execution of forward and

68 backward computations [35]. Instead, learning is realized through repetition of local and

69 asynchronous stochastic updates of states of neurons and synapses in a network. Because

70 the algorithm is not derived as an optimization of objective functions, it rarely exhibits

71 serious overfitting. We also discuss the close relationship between our algorithm and the

72 temporal difference (TD) learning [38] of reinforcement learning.

\section{Results}

\section{Neural networks}

76 Most connections between cortical neurons are redundantly realized by multiple synapses

77 [27, 39]. Introducing this redundancy and stochasticity of the neurons and synapses, we 78 model a neural network whose connections are all realized by multiple synapses. Within 79 this model, each neuron and synapse is represented by a binary stochastic variable (Fig. 80 1a, Methods). The value of a neural variable determines whether that neuron generates a 81 spike, whereas the value of a synaptic variable determines whether that synapse contacts 82 a dendrite of the postsynaptic neuron. A neuron in the network receives inputs from 83 presynaptic neurons and generates a spike with a probability that is a function of the sum 84 of these inputs (see Methods). External inputs, including the target outputs of supervised 85 learning, are presented to the network as variables for some neurons, namely visible 86 neurons, are fixed to values of these input variables. These input data, thus, should be 87 represented by binary vectors. Other neurons in the network, which do not receive 88 external data directly, are referred to as "hidden neurons".

\section{Biologically plausible learning}

91 With the fundamentals of the network as described above, we formulate learning in the 92 network as a continuing sampling of all the free variables, which include variables 93 representing all synapses and hidden neurons, in the network from a posterior distribution 94 conditioned on the external environment or a given dataset (see Methods). In other words, 95 we hypothesize that the stochastic dynamics of the neurons and synapses in the cortex 96 constitute a continuing random process that aimed at generating a network that suitably 97 interprets the external world.

99 A sampling from the posterior distribution is computationally and biologically intractable 
100 in general, due to the high dimensionality of the system and the complex dependencies

101 among its variables. To solve this problem, we hypothesize that the sampling in the cortex

102 is a Gibbs sampling [40]. The Gibbs sampling ensures that we can replace a sampling

103 from the high dimensional posterior distribution with iterative samplings of each variable

104 from a posterior distribution of that variable conditioned on all other variables.

105 Furthermore, due to the flexibility of the Gibbs sampling, each sampling can be

106 performed in any order and with any frequency. This implies that each neuron and

107 synapse can asynchronously and irregularly update their variables with their own

108 individually determined timings without any global schedule or coordination among

109 them.

110

111 Applying Bayes' theorem to the posterior distributions, we obtain stochastic dynamics,

112 i.e. stochastic update rules, for the neurons and the synapses (see Methods) as

$$
\begin{gathered}
P\left(x_{d i}=1 \mid \cdots\right)=\sigma\left(v_{d i}+b_{d i}\right) \\
b_{d i} \leftarrow\left(1-r_{b}\right) b_{d i}+r_{b} \sum_{j} w_{i j}\left(x_{d j}-\sigma\left(v_{d j}\right)\right)
\end{gathered}
$$

113 for a neuron and

$$
\begin{gathered}
P\left(s_{i j k}=1 \mid \cdots\right)=\sigma\left(a_{i j k} q_{i j}\right) \\
q_{i j} \leftarrow\left(1-r_{q}\right) q_{i j}+r_{q} \sum_{d} x_{d i}\left(x_{d j}-\sigma\left(v_{d j}\right)\right)
\end{gathered}
$$

114 for a synapse, where the dots represent all variables other than the target variable, $x_{d i}$ is

115 the state of the $i$ th neuron when the $d$ th datum is given to the network, $v_{d i}=\sum_{j} x_{j} w_{j i}$ is

116 the membrane potential of the neuron, $s_{i j k}$ is the state of the $k$ th synapse of the

117 connection form $i$ th neuron to the $j$ th neuron, $r_{b}$ and $r_{q}$ are constants which

118 characterize timescales of evolution of $b_{d i}$ and $q_{i j}$, respectively. $\sigma(x)$ is the sigmoidal

119 function. Following these equations, the state of each neuron and each synapse is

120 repeatedly updated. This simple repetition of irregular and asynchronous stochastic

121 updates is the learning algorithm of the neural network.

123 Unlike backpropagation learning, in which the alternating execution of forward and

124 backward computations is required, our algorithm realizes learning through the simple

125 iteration of a single computation for each variable. Furthermore, the equations are local in

126 the sense that they depend only on neurons and synapses directly connected to the 
127 updated variable, i.e. the Markov blanket of the variable. In addition, the variables do not

128 require any global signals, such as the error of the current output of the network. This

129 asynchronicity and local nature of the dynamics of the learning must be particularly

130 suited to biological implementation of the algorithm.

132 Note the difference between the summation indices in Eqs. (2) and (4). Synaptic update

133 requires summation over the all data of a given dataset, while neural update does not. This

134 difference is a result of the difference between the data dependencies of the variables

135 (Figure $1 \mathrm{~b}$ and 1c, see Methods for full details). Because of this difference, synapses need

136 to accumulate the neural activities for many, ideally all, data in the dataset before update

137 their states, while neurons reset their states independently in a manner that depends on

138 each datum individually. This implies that if data are provided sequentially to the network,

139 as in the brain, synapses must evolve much more slowly than neurons (Figure 1d). This

140 explains greatly different timescales of neurons and synapses in the brain. In the

141 following numerical simulations, however, we perform neural updates for all data of the

142 dataset in parallel to accelerates the computational speed.

\section{Synaptic plasticity}

145 The derived update rule for synapses given in Eqs. (3) and (4) yields plasticity similar to

146 that exhibited by cortical synapses [29]. Each term in the summation in Eq. (3) vanishes

147 unless the presynaptic neuron $x_{d i}$ fires, and if the neuron does fire, whether it will be

148 positive (LTP) or negative (LTD) depends on whether or not the postsynaptic neuron $x_{d j}$

149 fires simultaneously. For this reason, each synaptic weight increases if the network

150 receives many data in which the pre-synaptic and post-synaptic neurons of the connection

151 fire synchronously, while it decreases if the pre-synaptic and post-synaptic neurons fire

152 asynchronously (Fig. 1e).

154 Interestingly, the update rule depends on membrane potential, $v_{d j}$, in addition to the 155 spikes, $x_{d j}$, of the postsynaptic neuron. This is consistent with a recently proposed model 156 of STDP that accounts for various properties of synaptic plasticity by introducing the 157 average membrane potential of postsynaptic neurons into the model [30]. It is also 158 noteworthy that, unlike most existing models of synaptic plasticity, the plasticity derived 159 here does not require any artificial bound on synaptic weights. The incremental variation 
160 of each synaptic weight automatically decays to zero when the magnitude of the weight

161 becomes very large (Fig. 1e), because $x_{d j}-\sigma\left(v_{d j}\right)$ decays to zero in such cases.

\section{Retrograde modulation of excitability}

164 The term, $b_{d i}$, of the neural dynamics given in Eq. (2) represents the retrograde 165 modulation of the firing probability of a neuron by its postsynaptic neurons. This term 166 provides a stochastic variant of the error propagation in backpropagation learning. Recall 167 that a target output is given to the network by fixing the states of the corresponding visible 168 neurons to the desired output. Assume that the $j$ th neuron is one of these neurons. Then $169 x_{d j}$ gives the desired value, and the term $x_{d j}-\sigma\left(v_{d j}\right)$ in the bias $b_{d i}$ of the $i$ th neuron 170 gives the difference between the desired value and the expected value of $x_{d j}$ when the $171 j$ th neuron is not fixed, which is identical to the error in backpropagation learning when 172 the squared error is used as the loss function. Owing to the retrograde modulation, 173 information regarding the desired output provided only to output neurons can spread, i.e. 174 diffuse, over the entire network, even though the variables of the neurons are updated 175 independently, without coordinated scheduling of error backpropagation. As we will show later, unlike backpropagation learning, the retrograde modulation need not be immediately affected by spikes of the postsynaptic neurons but, rather, can slowly integrate the effects of the spikes. This means that the retrograde bias is determined by the average spike history of the postsynaptic neurons over a finite, presumably quite long, duration. Such slow modulation of the excitability of the neurons could be due to slow changes in the axon initial segment or a long-term modulation of the spike threshold. This seems biologically plausible, as it can be implemented in real cortical circuits. To our knowledge, experiments to verify the existence of such slow retrograde modulation of the excitability of neurons have not yet been attempted.

\section{Feedforward networks}

188 To understand how the algorithm works, we study its application to a simple problem of 189 supervised learning for a three-layered network (see Methods for full details). Figure 2a 190 displays the evolution of the training and test accuracies as functions of the number of the 191 sampling iteration. It is seen that these accuracies nearly coincide, and they quickly 192 increase to values close to unity and remain there. Significantly, even while these 
193 accuracies remain nearly constant, the synaptic weights of the network continue to

194 fluctuate greatly (Fig. 2b), and the firing patterns of the hidden neurons also continue to

195 change, even when the same datum is given to the network, without converging to a fixed

196 pattern (Fig. 2c). These results are consistent with experimental observations of

197 continuing fluctuations of synapses and the trial-to-trial variability of cortical neural

198 activity.

199

200 In order to study the robustness of the algorithm with respect to constants of the algorithm,

201 we calculated the realized test accuracies of the network after learning for various

202 combinations of values of the number of synapses per connection, $K$, and the maximum

203 amplitude of synapses, $a_{0}$, (Fig. 2d, see Methos). Except in a narrow range in which one

204 of these constants is so small that the possible maximum weight given by $a_{0} K$ is small,

205 the network almost perfectly learns to perform the task.

Next, we consider the influence of the parameters $r_{b}$ and $r_{q}$ (Figure 2e, 2f). Unless $r_{q}$ is extremely small, and hence $q$ evolves extremely slowly, the training and test accuracies will increase and reach values near unity, while their convergence speed decreases as $r_{b}$

210 or $r_{q}$ decreases. (Note that the synaptic evolution is slower than the neuronal evolution

211 even when $r_{q}=1$, as discussed above. Therefore, a small value of $r_{q}$ may result in

212 unrealistically slow synaptic dynamics.) We can thus conclude that the learning is not

213 practically hindered by the use of slow timescales of $b$.

215 We next study the application of the algorithm to training multilayered feedforward 216 networks using the MNIST dataset to demonstrate the applicability of the method to 217 practical problems (Fig. 3). We found that the accuracies quickly increase to values near $21895 \%$, while the number of required iterations and the asymptotically realized accuracies 219 decrease slightly as we increase the number of layers in the network (Fig. 3a-c). Figure 3d 220 displays examples of numerals that the network fails to recognize. These are quite 221 ambiguous and difficult even for a human to identify with confidence.

223 Figure $3 \mathrm{e}$ illustrates examples of connection weights from the input to the hidden neurons 224 in a three-layered network after training. These correspond to the receptive fields of the 225 hidden neurons. We can see that Gabor-filter-like localized structures that resemble 
226 receptive fields of neurons in the primary visual cortex [32] are often organized through

227 the learning. The introduction of a sparse constraint on neural activity into the loss

228 function of learning is known to provide Gabor-filter like receptive fields for an artificial

229 neural network [33]. It is interesting that the network can acquire similar localized

230 structures of receptive fields even without any explicit additional constraint on the

231 learning algorithm.

233 The learning algorithm can avoid serious overfitting to the training data because it is not

234 derived as a direct optimization of any objective functions. To see this point, we trained a

235 three-layered feedforward network using small numbers of samples of the MNIST dataset,

236 and compared the resulting training and test accuracies with those obtained from

237 backpropagation learning using the stochastic gradient descent (SGD) and ADAM

238 algorithms [41] (Fig. 3f). We found that for both the SGD and ADAM algorithms, as the

239 size of the training dataset is increased, the training accuracy decreases (in the SGD case)

240 or remain nearly constant (in the ADAM case), while the test accuracy increases

241 monotonically, which implies overfitting to the training dataset. Contrastingly, with the

242 proposed algorithm, as the size of the training dataset is increased, both the training

243 accuracy and the test accuracy increase, maintaining a slight difference between them.

244 This implies that the serious overfitting does not occur in the proposed learning 245 algorithm.

\section{Most efficient power-law coding}

248 A recent experiment carried out by simultaneously recording the activity of a very large

249 number of neurons revealed that the variance spectrum of the principal component of 250 neural activities obeys a power law with an exponent -1.04 that is slightly less than -1 [7].

251 The authors of the paper [7] proved that if the exponent is greater than -1, the population 252 code by the neurons could not be smooth, while if the exponent is less than -1 , high 253 dimensionality of the population code is not fully realized. Thus, the experimentally 254 observed power-law coding with an exponent slightly less than -1 is the most efficient in 255 the sense that in this case, the population response of the neurons lies on a manifold of the 256 highest possible dimension while maintaining high generalizability.

258 To test whether a network trained by the proposed algorithm realizes the most efficient 
259 coding, we numerically calculated the variance spectrum of the principle components of

260 the mean activity of the neurons in the hidden layer of a network trained with the MNIST

261 dataset. As shown in Fig. 4a, the variance spectrum exhibits clear power-law decay with

262 an exponent of -1.06 . This is very close to the experimental result, and is indeed slightly

263 less than -1 . We conclude that the learning algorithm leads the network to the most

264 efficient coding.

265

266 We next study how the exponent of the power law develops during learning. Figure $4 \mathrm{~b}$

267 shows that the exponent approaches a value close to -1 from below as the learning

268 proceeds. This result implies that the network first learns a coarse representation of the

269 dataset and then gradually acquires finer structures while maintaining generalizability of

270 the representation of the data in coding space. This leads us to conclude that the

271 robustness or generalizability of the population coding takes priority over the precision of

272 the data representation in the learning. This priority must particularly be beneficial for

273 animals that must survive in a ceaselessly changing environment.

\section{Recurrent networks}

276 We next applied the algorithm to train a network with recurrent connections (Fig. 5a)

277 using the MNIST dataset. Figure 5b displays the evolution of the training and test

278 accuracies as functions of the number of sampling iterations. The accuracies were

279 obtained from the states of the output neurons of the network measured after recursive

280 evolutions of the states of the hidden neurons. We see that, as in the case of the results for

281 the feedforward networks, the accuracies nearly coincide and rapidly increase. This

282 implies that the algorithm is even able to train a recurrent network and rarely overfits.

\section{Statistics of network motifs}

285 It has been reported that local cortical circuits are highly nonrandom, and that 286 connectivity patterns consisting of multiple neurons, known as network motifs, exhibit a 287 characteristic distribution in which highly clustered patterns are overrepresented [31]. To 288 study whether a recurrent network trained by the proposed algorithm acquires a similar 289 distribution of connectivity patterns, we determined connectivity of triplets of neurons in 290 a trained recurrent network. The statistics for the ratio of the actual counts of triplet 291 patterns to the chance level are plotted in Figure 5c. The same ratios for the experimental 
292 results are also overlaid in the figure. While there are some exceptional cases in which the

293 ratios obtained here are somewhat larger than those obtained experimentally, the two

294 distributions of triplet patterns are surprisingly similar. As observed experimentally,

295 highly-connected motifs, i.e., those numbered 10 through 16 in Fig. 5c, are

296 overrepresented by a factor several times greater than chance level. These results support

297 the validity of the derived algorithm as a model describing the formation of local cortical

298 circuits.

\section{Connection weights and receptive field correlation}

301 A recent experiment of the primary visual cortex revealed that the connection weights

302 between pairs of pyramidal neurons become stronger as the receptive fields become more

303 similar [34]. To test whether the trained recurrent network accounts for this relationship,

304 we measured the connection weights between pairs of neurons and the receptive field

305 correlations between these neurons (fig. 6a). We found that the average connection

306 weight between neurons is positively correlated with the correlation between the

307 receptive fields of these neurons (Fig. 6b). Particularly, by restricting our analysis to only

308 connection weights with positive values (i.e., $w_{i j}>0$ ), we were able to reproduce a

309 nonlinear relationship between the average connection weight and the receptive field

310 correlation (Fig. 6c), which was similar with the experimental result [34].

\section{Temporal sequence learning}

313 We next consider the application of the algorithm to train recurrent networks with

314 temporal sequences (Fig. 7). We prepared periodic temporal sequences in which the same

315 temporal inputs may appear multiple times at different times, and trained networks to

316 predict the next input of the current sequence. In this case, networks need to learn to store

317 the history of inputs over some interval to generate the desired output. The training

318 procedure was the same as that used in the case considered in Figs. 2-5, except that we

319 identified the iteration of the updates of the variables of the network as the time

320 development. In contrast to the algorithm known as "backpropagation through time", this

321 procedure does not require virtually unfolding the recurrent connections of the network

322 along the time axis.

323

324 We prepared two sequences that require one-step and two-step memories, respectively. 
325 For both tasks, the networks were successfully trained to output the desired sequences by

326 the learning algorithm. We find that after learning, the output produced by the network in

327 any given case depends not only on the current input but also on past inputs. This

328 indicates that, through the learning, the algorithm causes the network to store input

329 histories into the activity of the hidden neurons.

\section{Discussion}

332 In this study, we showed that Gibbs sampling from a joint posterior distribution of

333 neurons and synapses in a network conditioned on an external environment or given

334 dataset explains the stochastic natures of synaptic development and neural activities of

335 cortical circuits. This provides a practical and biologically plausible learning algorithm

336 that yields results consistent with various experimental findings for cortical circuits. The

337 derived stochastic dynamics of synapses are consistent with the plasticity of cortical

338 synapses, and those of neurons naturally describe highly irregular features and the

339 trial-to-trial variability of spike trains of cortical neurons.

341 The evolution equation for neurons has a term that results in the retrograde modulation of

342 the excitability of a neuron by its postsynaptic neurons. Due to this term, the algorithm

343 acts as a stochastic variant of algorithms with error backpropagation through which target

344 outputs provided to a part of network can spread over the entire network. However, in

345 contrast to the case of backpropagation learning, the retrograde modulation seen in our

346 model requires neither synchronous nor precisely coordinated operations, which are

347 major reason that backpropagation has not been regarded as the learning principle of the

348 brain. Retrograde modulation need not be immediately affected by postsynaptic action

349 potentials but, rather, can slowly integrate postsynaptic spikes. The mechanisms regarded

350 as likely to be responsible for this behavior include the combination of action potential

351 backpropagation from soma to dendritic spines [42] and retrograde transsynaptic

352 transport of certain chemicals [43], glia-mediated modulation [44], and disynaptic

353 connections from postsynaptic to presynaptic neurons. Experimental confirmation of this

354 modulation would be convincing evidence to support the validity of the proposed

355 framework as a learning and computational principle of the brain.

357 The reason why both neurons and synapses must be stochastic in the cortex can be 
358 understood as follows. Suppose that the primary purpose of the cortex is to generate

359 synaptic weights that are consistent with the external environment. Mathematically, this

360 can be formulated by generating samples of synaptic weights from a Bayesian posterior

361 distribution conditioned on the external environment, which is derived from the

362 likelihood function of the synaptic states determined by the dataset. However, when the

363 network has hidden neurons, the likelihood is given by a marginal distribution of the joint

364 conditional distribution of hidden neurons and synapses in which neural and synaptic

365 variables are strongly coupled. Thus, sampling of synaptic states inevitably also requires

366 sampling of neural states. In this way, the stochastic behaviors of neurons and synapses

367 are integrated in the cortical dynamics.

369 It has been pointed out that the temporal difference (TD) method of reinforcement

370 learning provides a model of spike-timing-dependent Hebbian plasticity [38]. In TD

371 learning, the value function of the current state is updated after the delivery of a reward

372 such that the value function includes the TD error defined as the difference between the

373 value of the delivered reward and its predicted value. Interestingly, each term in the

374 evolution equations of the latent variables of the synapses and neurons, i.e. $b_{d i}$ and $q_{i j}$,

375 can be regarded as a representation of the TD error of the delivered spikes instead of the

376 rewards. Indeed, each term in Equations (2) and (4) takes the form of a scaled difference

377 between the measured spike of a postsynaptic neuron, $x_{d j}$, and its expected value,

$378 \sigma\left(v_{d j}\right)$, before observation of the spike. In this analogy, therefore, each spike plays the

379 role of a reward for the presynaptic neurons and synapses from these neurons. At the

380 same time, each spike is also transmitted to postsynaptic neurons, thereby changing their

381 firing probabilities in a manner that depends on the corresponding synaptic weights. Thus,

382 each spike in the cortex plays two roles, rewarding backward units and selecting next

383 states by triggering the next spikes. It will be a fascinating topic of future research to

384 theoretically elucidate the relationship between our algorithm and TD learning.

386 The algorithm developed here is closely related to the Boltzmann machine [37], which is

387 a stochastic recurrent neural network in which neurons are represented by stochastic

388 binary variables and iteratively updated to realize a thermal equilibrium state of a

389 globally defined energy function specified by a given temperature. In that algorithm,

390 symmetric connection weights between neurons are trained with a gradient descent 
391 method to make the equilibrium state approximate a target distribution in which the

392 neural states of given a dataset have high probability. The algorithm developed in the

393 work can be regarded as an extension of the Boltzmann machine in which the Bayesian

394 posterior distribution is directly considered, instead of a thermal equilibrium of an energy

395 function, and in which synapses in addition to neurons are modeled as stochastic

396 variables to be sampled. This extension results in biologically realistic update rules of

397 variables that are implemented concurrently for neurons and synapses with no need for

398 explicit switching of different computations or fine scheduling of parameters, such as

399 simulated annealing.

401 In the limit of a large number of training data, the posterior distribution of Bayesian

402 inference converges to a delta function whose peak position coincides with the result of

403 the maximum likelihood estimation (see Methods). Therefore, if a sufficiently large

404 amount of external data is provided to the network, our learning algorithm almost surely

405 generates synaptic and neural variables that most suitably reflect the external data. This

406 situation contrasts with that for backpropagation learning, in which convergence to an

407 optimal solution is not always guaranteed, even when a large amount of data is used.

409 Because it seems to operate in accordance with a basic principle of neural computation

410 and learning, we believe that our model provides a theoretical foundation for various

411 experimental findings regarding cortical dynamics and various methods of machine

412 learning. Most of the recent experimental findings regarding neuroscience have not yet

413 been fully utilized in the development of machine learning. This may be because

414 backpropagation learning is not consistent with the functioning of the brain. These

415 experimental results include results regarding short-term plasticity of synapses [45],

416 Dale's principle, the long-tail distribution of synaptic weights [31], columnar structure,

417 laminar organization, canonical circuits [46, 47], and innate structure formed through

418 developmental stages [48]. Incorporating these features into our model with the goal of

419 clarifying their functional roles is an important future project. Introducing precise

420 continuous dynamics of membrane potentials and spike generation mechanisms into the

421 model are also important topic to be investigated.

\section{References}


424 [1] Shadlen, M. N., \& Newsome, W. T. (1994). Noise, neural codes and cortical 425 organization. Current Opinion in Neurobiology, 4(4), 569-579.

426 [2] Arieli, A., Sterkin, A., Grinvald, A., \& Aertsen, A. (1996). Dynamics of ongoing 427 activity: Explanation of the large variability in evoked cortical responses. Science, 428 273(5283), 1868-1871.

429 [3] Kenet, T., Bibitchkov, D., Tsodyks, M., Grinvald, A., \& Arieli, A. (2003).

430 Spontaneously emerging cortical representations of visual attributes. Nature, 425(6961), $431954-956$.

432 [4] Destexhe, A., Rudolph, M., \& Paré, D. (2003). The high-conductance state of 433 neocortical neurons in vivo. Nature Reviews Neuroscience, 4(9), 739-751.

434 [5] Berkes, P., Orbán, G., Lengyel, M., \& Fiser, J. (2011). Spontaneous cortical activity 435 reveals hallmarks of an optimal internal model of the environment. Science, 331(6013), $43683-87$.

437 [6] Pouget, A., Beck, J. M., Ma, W. J., \& Latham, P. E. (2013). Probabilistic brains: 438 knowns and unknowns. Nature Neuroscience, 16(9), 1170-1178.

439 [7] Stringer, C., Pachitariu, M., Steinmetz, N., Carandini, M., \& Harris, K. D. (2019).

440 High-dimensional geometry of population responses in visual cortex. Nature, 571(7765), $441361-365$.

442 [8] Llera-Montero, M., Sacramento, J., \& Costa, R. P. (2019). Computational roles of 443 plastic probabilistic synapses. Current Opinion in Neurobiology, 54, 90-97.

444 [9] Stettler, D. D., Yamahachi, H., Li, W., Denk, W., \& Gilbert, C. D. (2006). Axons and 445 Synaptic Boutons Are Highly Dynamic in Adult Visual Cortex. Neuron, 49(6), 877-887.

446 [10] Yasumatsu, N., Matsuzaki, M., Miyazaki, T., Noguchi, J., \& Kasai, H. (2008).

447 Principles of long-term dynamics of dendritic spines. The Journal of Neuroscience, 448 28(50), 13592-13608.

449 [11] Holtmaat, A., \& Svoboda, K. (2009). Experience-dependent structural synaptic 450 plasticity in the mammalian brain. Nature Reviews Neuroscience, 10(9), 647-658.

451 [12] Chen, X., Leischner, U., Rochefort, N. L., Nelken, I., \& Konnerth, A. (2011).

452 Functional mapping of single spines in cortical neurons in vivo. Nature, 475(7357), 453 501-505.

454 [13] Attardo, A., Fitzgerald, J. E., \& Schnitzer, M. J. (2015). Impermanence of dendritic 455 spines in live adult CA1 hippocampus. Nature, 523(7562), 592-596.

456 [14] Mongillo, G., Rumpel, S., \& Loewenstein, Y. (2017). Intrinsic volatility of synaptic 
457 connections - a challenge to the synaptic trace theory of memory. Current Opinion in

458 Neurobiology, 46, 7-13.

459 [15] Pascoli, V., Hiver, A., Van Zessen, R., Loureiro, M., Achargui, R., Harada, M., et al.

460 (2018). Stochastic synaptic plasticity underlying compulsion in a model of addiction.

461 Nature, 564(7736), 366-371.

462 [16] Allen, C., \& Stevens, C. F. (1994). An evaluation of causes for unreliability of

463 synaptic transmission. Proceedings of the National Academy of Sciences, 91(22),

464 10380-10383.

465 [17] Katz, L. C., \& Shatz, C. J. (1996). Synaptic Activity and the Construction of Cortical

466 Circuits. Science, 274(5290), 1133-1138.

467 [18] Branco, T., \& Staras, K. (2009). The probability of neurotransmitter release:

468 variability and feedback control at single synapses. Nature Reviews Neuroscience, 10(5),

$469373-383$.

470 [19] Deneve, S., Latham, P. E., \& Pouget, A. (2001). Efficient computation and cue

471 integration with noisy population codes. Nature Neuroscience, 4(8), 826-831.

472 [20] Ma, W. J., Beck, J. M., Latham, P. E., \& Pouget, A. (2006). Bayesian inference with

473 probabilistic population codes. Nature Neuroscience, 9(11), 1432-1438.

474 [21] Doya, K., Ishii, S., Pouget, A., \& Rao, R. P. (Eds.). (2007). Bayesian brain:

475 Probabilistic approaches to neural coding. MIT press.

476 [22] Soltani, A., \& Wang, X.-J. (2010). Synaptic computation underlying probabilistic

477 inference. Nature Neuroscience, 13(1), 112-119.

478 [23] Kappel, D., Habenschuss, S., Legenstein, R., \& Maass, W. (2015). Network

479 Plasticity as Bayesian Inference. PLoS Computational Biology, 11(11), e1004485.

480 [24] Aitchison, L., \& Latham, P. E. (2015). Synaptic sampling: A connection between

481 PSP variability and uncertainty explains neurophysiological observations. arXiv preprint 482 arXiv:1505.04544.

483 [25] Orbán, G., Berkes, P., Fiser, J., \& Lengyel, M. (2016). Neural Variability and

484 Sampling-Based Probabilistic Representations in the Visual Cortex. Neuron, 92(2), 485 530-543.

486 [26] Neftci, E. O., Pedroni, B. U., Joshi, S., Al-Shedivat, M., \& Cauwenberghs, G. (2016).

487 Stochastic Synapses Enable Efficient Brain-Inspired Learning Machines. Frontiers in 488 Neuroscience, 10(99), 796.

489 [27] Hiratani, N., \& Fukai, T. (2018). Redundancy in synaptic connections enables 
490 neurons to learn optimally. Proceedings of the National Academy of Sciences of the

491 United States of America, 115(29), E6871-E6879.

492 [28] Baldassi, C., Gerace, F., Kappen, H. J., Lucibello, C., Saglietti, L., Tartaglione, E., \&

493 Zecchina, R. (2018). Role of Synaptic Stochasticity in Training Low-Precision Neural

494 Networks. Physical Review Letters, 120(26), 268103.

495 [29] Bi, G. Q., \& Poo, M. M. (1998). Synaptic modifications in cultured hippocampal

496 neurons: dependence on spike timing, synaptic strength, and postsynaptic cell type. The

497 Journal of Neuroscience, 18(24), 10464-10472.

498 [30] Clopath, C., Büsing, L., Vasilaki, E., \& Gerstner, W. (2010). Connectivity reflects

499 coding: a model of voltage-based STDP with homeostasis. Nature Neuroscience, 13(3),

$500 \quad 344-352$.

501 [31] Song, S., Sjöström, P. J., Reigl, M., Nelson, S. B., \& Chklovskii, D. B. (2005).

502 Highly nonrandom features of synaptic connectivity in local cortical circuits. PLoS

503 Biology, 3(3), e68.

504 [32] Jones, J. P., \& Palmer, L. A. (1987). An evaluation of the two-dimensional Gabor

505 filter model of simple receptive fields in cat striate cortex. Journal of neurophysiology,

506 58(6), 1233-1258.

507 [33] Olshausen, B. A., \& Field, D. J. (1996). Emergence of simple-cell receptive field

508 properties by learning a sparse code for natural images. Nature, 381(6583), 607-609.

509 [34] Cossell, L., Iacaruso, M. F., Muir, D. R., Houlton, R., Sader, E. N., Ko, H., et al.

510 (2015). Functional organization of excitatory synaptic strength in primary visual cortex.

511 Nature, 518(7539), 399-403.

512 [35] Bengio, Y., Lee, D.-H., Bornschein, J., Mesnard, T., \& Lin, Z. (2015). Towards

513 Biologically Plausible Deep Learning. arXiv.org.

514 [36] Lillicrap, T. P., Cownden, D., Tweed, D. B., \& Akerman, C. J. (2016). Random

515 synaptic feedback weights support error backpropagation for deep learning. Nature

516 Communications, 7, ncomms 13276.

517 [37] Ackley, D., Hinton, G., and Sejnowski, T. (1985). A Learning Algorithm for

518 Boltzmann Machines. Cognitive Science, 9(1):147-169.

519 [38] Rao, R. P. N., \& Sejnowski, T. J. (2001). Spike-timing-dependent Hebbian plasticity

520 as temporal difference learning. Neural Computation, 13(10), 2221-2237.

521 [39] Feldmeyer, D., Egger, V., Lübke, J., \& Sakmann, B. (1999). Reliable synaptic

522 connections between pairs of excitatory layer 4 neurones within a single "barrel" of 
523 developing rat somatosensory cortex. The Journal of Physiology, 521(1), 169-190.

524 [40] Geman, S.; Geman, D. (1984). "Stochastic Relaxation, Gibbs Distributions, and the

525 Bayesian Restoration of Images". IEEE Transactions on Pattern Analysis and Machine

526 Intelligence. 6 (6): 721-741.

527 [41] Kingma, D. P., \& Ba, J. (2014). Adam: A method for stochastic optimization. arXiv

528 preprint arXiv:1412.6980.

529 [42] Stuart, G., Spruston, N., Sakmann, B., \& Häusser, M. (1997). Action potential

530 initiation and backpropagation in neurons of the mammalian CNS. Trends in

531 Neurosciences, 20(3), 125-131.

532 [43] Regehr, W. G., Carey, M. R., \& Best, A. R. (2009). Activity-Dependent Regulation

533 of Synapses by Retrograde Messengers. Neuron, 63(2), 154-170.

534 [44] Fields, R. D., \& Stevens-Graham, B. (2002). New insights into neuron-glia

535 communication. Science, 298(5593), 556-562.

536 [45] Markram, H. and Tsodyks, M. (1996). Redistribution of synaptic efficacy between

537 neocortical pyramidal neurons. Nature. 382(6594): 807-810.

538 [46] Silberberg, G., Grillner, S., LeBeau, F. E. N., Maex, R., \& Markram, H. (2005).

539 Synaptic pathways in neural microcircuits. Trends in Neurosciences, 28(10), 541-551.

540 [47] Bastos, A. M., Usrey, W. M., Adams, R. A., Mangun, G. R., Fries, P., \& Friston, K. J.

541 (2012). Canonical Microcircuits for Predictive Coding. Neuron, 76(4), 695-711.

542 [48] Ullman, S. (2019). Using neuroscience to develop artificial intelligence. Science,

543 363(6428), 692-693.

\section{Figure captions}

\section{Figure 1}

547 Learning as a Gibbs sampling of synapses and neurons. (a) A neural network is modeled

548 as a population of neurons connected to each other via multiple synapses. (b) A simple

549 neural network consists of three neurons and four synapses per connection. The input,

550 hidden, and output neurons are denoted by $x_{0}, x_{1}$ and $x_{2}$, respectively. (c) A graphical

551 model representation of the network shown in (b) in the case that a dataset consisting of D

552 data is presented to the network. Note that the synaptic variables are shared by all data in

553 the dataset while neural variables are not. The white and gray circles represent free and

554 fixed variables, respectively. (d) Schematic of stochastic evolution of neural and synaptic

555 variables. Synapses must evolve much more slowly than neurons to allow the network to 
556 incorporate many, ideally all, data in the dataset. Note that values of the visible neurons,

$557 x_{0}, x_{1}$, and $x_{5}$ in the figure, are fixed to each datum in the given dataset. (e) Evolution of

558 a synaptic weight (right panel) when presynaptic and postsynaptic neurons fire (left

559 panel) synchronously (cyan) and asynchronously (magenta).

\section{Figure 2}

562 Supervised learning of feed-forward networks using a simple artificial dataset. (a)

563 Training (cyan) and test (magenta) accuracies of a three-layered network as functions of

564 the number of sampling iterations (details given in Methods). (b) Evolution of randomly

565 chosen synaptic weights of the network. Different colors are used for different

566 presynaptic neurons. (c) Evolution of the states of randomly chosen hidden neurons when

567 the same datum in the dataset is presented to the network. (d) Test accuracies realized by

568 the learning algorithm with various values of the number of synapses per connection, $K$,

569 and maximum amplitude of a synapse, $a_{0}$. (e) Evolution of accuracies for $r_{b}=$

$5701.0,0.1,0.01,0.001$, from top to bottom. (f) The same as (i) but for

$571 r_{q}=1.0,0.1,0.01,0.001$, from top to bottom.

572

\section{Figure 3}

574 Supervised learning of feed-forward networks using the MNIST dataset. (a-c) Training 575 accuracies (cyan) and test accuracies (magenta) as functions of the number of sampling

576 iterations for (a) three-, (b) four-, and (c) five-layered networks. Each number in a circle

577 indicates the number of neurons in the corresponding layer. (d) Examples of training

578 images (upper row) and test images (lower low) that the three-layered network fails to

579 recognize. (e) Examples of receptive fields of hidden neurons in the three-layered

580 network. (f) Realized accuracies of the three-layered network as functions of the size of

581 the training dataset. The network was trained by the proposed algorithm (left panel), a

582 backpropagation learning algorithm with a naïve stochastic gradient descendent (middle

583 panel), and with the ADAM algorithm (right panel).

\section{Figure 4}

586 Nearly optimal power-law decay of the variance spectrum for the principal component of

587 the neural activity. (a) Variance spectrum of the principle components of the mean

588 activity of the hidden neurons of the three-layered network after training (cyan). The 
589 variances are arranged in descending order. The line (magenta) indicates the critical slope

590 corresponding to an exponent of -1. (b) Evolution of the power-law exponent of the

591 variance spectrum as a function of the number of sampling iterations.

\section{Figure 5}

594 Supervised learning of a recurrent network using the MNIST dataset. (a) A recurrent

595 neural network. Each number in a circle indicates the number of neurons in the 596 corresponding layer. (b) Evolution of the training accuracies (cyan) and test accuracies

597 (magenta) of the recurrent network. (c) Average ratios of the actual numbers of 598 three-neuron patterns in the trained recurrent network to those predicted by the null 599 hypothesis (gray bars), (details given in Methods). The error bars indicate the range of $600 \pm 2 \sigma$. The green circles are experimental results for real cortical circuit [31].

\section{Figure 6}

603 Correlation between connection weights between pairs of neurons and the similarity of 604 the receptive fields of these neurons. (a) Correlation coefficients, $c_{i j}$, of the receptive 605 fields between pairs of neurons and connection weights, $w_{i j}$, between these neurons are 606 measured for the recurrent network after training. (b) The connection weights between 607 pairs of neurons positively correlates with the receptive field correlations between these 608 neurons. (c) The average weight of these connections with positive weights, $w_{i j}>0$, as 609 a function of the receptive field correlation. Underlying histogram shows the distribution 610 of the receptive field correlations for the pairs of neurons. These results correspond to 611 Figure $2 \mathrm{~g}$ and $2 \mathrm{i}$ of [34].

\section{$613 \quad$ Figure 7}

614 Supervised learning of recurrent networks using temporal sequences. (a) Network 615 structure. (b) Input sequences (upper panels) and target output sequences (lower panels) 616 of the first dataset. The asterisks indicate examples of times at which the network must 617 output different patterns while the current input to the network is the same. (c) Activities 618 of hidden neurons (upper panels) and output neurons (lower panels) after 0, 80, and 400 619 sampling iterations. (d) Input sequences (upper panels) and target output sequences 620 (lower panels) of the second dataset. (e) The same as (c), but for the second dataset. 


\section{Methods}

\section{Neural networks}

624 Introducing the redundancy and stochasticity of the neurons and synapses, we model a 625 neural network that consists of $\mathrm{N}$ neurons connected via $\mathrm{K}$ synapses per connection. The 626 connection weight from the $i$ th to the $j$ th neuron is given as a weighted sum of the 627 synaptic states as $w_{i j}=\sum_{k=1}^{K} s_{i j k} a_{i j k}$, where $s_{i j k} \in\{0,1\}$ is a binary random variable 628 describing the state of the $k$ th synapse of the connection from the $i$ th to the $j$ th neuron. 629 The weights are generally asymmetric, and we set $w_{i i}=0$ to avoid self-connections. 630 The strength, or amplitude, of a synapse, denoted by $a_{i j k}$, is a constant that represents the 631 contribution of the synapse to the weight, which can be interpreted as corresponding to 632 the amplitude of the miniature postsynaptic potential (PSP) of the synaptic contact. Note 633 that $a_{i j k}$ is a constant, and it is fixed during learning. For simplicity, we used an evenly 634 spaced sequence from $-a_{0}$ to $a_{0}$ for the values of the amplitude throughout the work: $635 a_{i j k}=a_{k}=(2(k-1) / K-1) a_{0}(k=1,2, \cdots, K)$.

The $i$ th neuron receives inputs from its presynaptic neurons and randomly generates a spike with the probability $P\left(x_{i}=1\right)=\sigma\left(v_{i}\right)=\sigma\left(\sum_{j} x_{j} w_{i j}\right)$, where the state of the neuron, $x_{i} \in\{0,1\}$, is a random binary variable representing the spike firing of the neuron, and $\sigma(x)$ is the activation function of a neuron, for which we use the sigmoidal

641 function $\sigma(x)=\left(1+e^{-x}\right)^{-x}$ throughout the paper. The weighted sum of inputs to the 642 neuron $v_{i}$ corresponds to the membrane potential of the neuron.

644 Neurons in the network are classified into three groups, input, output, and hidden. Input 645 and output neurons together are referred to as visible neurons. External data, including 646 the target outputs of supervised learning, are input into the network by fixing the states of 647 visible neurons to the values of the data. Each datum, therefore, must be a binary vector. 648 When we obtain the output of the network after and during learning, we fix only input 649 neurons, keeping output and hidden neurons free.

651 The states of the neurons, including the hidden and visible neurons, are updated in 652 response to each datum in the dataset as it is input, while the state of a synapse depends on 653 the dataset as a whole, because the aim of the learning is generally to obtain networks, i.e., 654 sets of synaptic states, that consistently reflect all of the data in the dataset (Figure 1c). 
655 For this reason, we write the state of the $i$ th neuron at the time that the network is

656 receiving the $d$ th datum of the dataset as $x_{d i}$, using the data index, while the state of the

657 synapse, $s_{i j k}$, does not have a data index. Figure 1c presents a graphical model

658 representation of the data dependency of the variables for the simplest case of a

659 three-layered neural network.

660

\section{Learning algorithm}

662 The learning of the network is modeled as a Gibbs sampling of all free variables from 663 their posterior joint distribution conditioned on the fixed variables, $664 P\left(\left\{x_{d i}\right\}_{i \in H, d \in D},\left\{s_{i j k}\right\}_{i, j \in N, k \in K} \mid\left\{x_{d i}\right\}_{i \in V}\right)$. Here, $d$ is the data index of the given dataset,

$665 D$, while $V$ and $H$ denote the sets of visible and hidden neurons, $N$ represents the set of 666 all neurons, and $K$ denotes the set of synapses for each connection. (The number of 667 variables of the network is thus $N D+W K$, where $W$ is the number of connections in 668 the network.) The Gibbs sampling allows us to replace sampling from a generally 669 high-dimensional joint distribution with a repetition of samplings of each single variable 670 from a posterior distribution conditioned on all other variables,

$$
P\left(x_{d i} \mid\left\{x_{d j}\right\}_{j \neq i},\left\{s_{i j k}\right\}\right)
$$

671 and

$$
P\left(s_{i j k} \mid\left\{x_{d i}\right\},\left\{s_{l m n}\right\}_{l m n \neq i j k}\right),
$$

672 for a neuron and a synapse respectively. In the Gibbs sampling, the order of the samplings 673 need not be fixed, but can be random. Also, the sampling frequencies of different 674 variables can be different. Therefore, in general, the state of each neuron or synapse will 675 change at times that are determined independently for each, depending only on the 676 conditions experienced individually by that neuron or synapse.

678 To derive an explicit description of the posterior distribution of a neuron, let us consider 679 the $\log$ likelihood ratio for $x_{d i}$. Using the Bayes rule, we obtain

$$
\log \frac{P\left(x_{d i}=1 \mid \cdots\right)}{1-P\left(x_{d i}=1 \mid \cdots\right)}=\log \frac{P\left(x_{d i}=1 \mid \cdots\right)}{P\left(x_{d i}=0 \mid \cdots\right)}
$$




$$
\begin{aligned}
& =\log \frac{P\left(x_{d i}=1\right)}{P\left(x_{d i}=0\right)} \prod_{j} \frac{P\left(x_{d j} \mid x_{d i}=0, \cdots\right)}{P\left(x_{d j} \mid x_{d i}=1, \cdots\right)} \\
& =\frac{\sigma\left(v_{d i}\right)}{1-\sigma\left(v_{d i}\right)} \\
& +\sum_{j}\left\{\log \left(1-\sigma\left(v_{d j,-i}+w_{i j}\right)\right)-\log \left(1-\sigma\left(v_{d j,-i}\right)\right), \quad x_{d j}=0\right.
\end{aligned}
$$

680 where the dots represent all variables other than $x_{d i}$, i.e., $\left\{x_{d j}\right\}_{j \neq i}$ and $\left\{s_{i j k}\right\}$, and we

681 have $v_{d j,-i}=\sum_{k \neq i} x_{d k} w_{k j}$. To obtain the 5th line, we have assumed that $v_{d j,-i} \gg w_{i j}$

682 and linearized each term of the summation with respect to $w_{i j}$. Solving the above

683 equation for $p\left(x_{d i}=1 \mid \cdots\right)$, we obtain Eq. (1) in the main text with

$$
b_{d i}=\sum_{j} w_{i j}\left(x_{d j}-\sigma\left(v_{d j}\right)\right)
$$

684 as the posterior distribution (i.e. the stochastic update rule) of the neuron.

685

686 Similarly, the log likelihood ratio for the synapse $s_{i j k}$ is

$$
\begin{aligned}
& \log \frac{P\left(s_{i j k}=1 \mid \cdots\right)}{1-P\left(s_{i j k}=1 \mid \cdots\right)}=\log \frac{P\left(s_{i j k}=1 \mid \cdots\right)}{P\left(s_{i j k}=0 \mid \cdots\right)} \\
& =\log \frac{P\left(s_{i j k}=1\right)}{P\left(s_{i j k}=0\right)} \prod_{d} \frac{P\left(x_{d j} \mid s_{i j k}=0, \cdots\right)}{P\left(x_{d j} \mid s_{i j k}=1, \cdots\right)} \\
& =q_{0, i j k} \\
& +\sum_{d}\left\{\begin{array}{c}
\log \left(1-\sigma\left(v_{d j,-i k}+s_{d i} a_{i j k}\right)\right)-\log \left(1-\sigma\left(v_{d j,-i k}\right)\right), \quad x_{d j}=0 \\
=q_{0, i j k}+\sum_{d}\left\{\begin{array}{c}
\left(1-\sigma\left(v_{d j}\right)\right) x_{d i} a_{i j k}, \quad x_{d j}=1 \\
-\sigma\left(v_{d j}\right) x_{d i} a_{i j k}, \quad x_{d j}=0
\end{array}\right.
\end{array}\right.
\end{aligned}
$$




$$
=q_{0, i j k}+a_{i j k} \sum_{j} x_{d i}\left(x_{d j}-\sigma\left(v_{d j}\right)\right),
$$

687 where the dots represent $\left\{x_{d i}\right\}$ and $\left\{s_{l m n}\right\}_{l m n \neq i j k}$, and we have $v_{d j,-i k}=\sum_{l} x_{l} w_{l j}-$ $688 x_{d i} s_{i j k} a_{i j k}$. To obtain the 5th line, we have assumed $v_{d j,-i k} \gg a_{i j k}$, and approximated 689 each term in the summation as a quantity linear in $a_{i j k}$. The constant $q_{0, i j k}$ represents 690 the log likelihood ratio of the prior distribution, $P\left(s_{i j k}\right)$, which simply vanishes unless 691 the prior distribution is biased. We assumed $q_{0, i j k}=0$ throughout the work. Solving the 692 above equation for $P\left(s_{i j k}=1 \mid \cdots\right)$ gives Eq. (3) in the main text and

$$
q_{i j}=\sum_{d} x_{d i}\left(x_{d j}-\sigma\left(v_{d j}\right)\right),
$$

693 which constitute the explicit description of the posterior distribution or the update rule of 694 the synapse.

696 Equations (5) implies that a spike of a neuron immediately changes $b_{d i}$ and excitability 697 of presynaptic neurons. However, such immediate retrograde modulation has not been 698 experimentally reported, and seems biologically implausible. Rather, it is biologically 699 more natural that $b_{d i}$ evolves slowly while accumulating the effects of postsynaptic 700 spikes as Eq. (3) in the main text, where $r_{b}$ characterizes the timescale of the evolution. 701 (Here, $r_{b}$ satisfies $0<r_{b} \leq 1$, while in the case $r_{b}=1$, Eq. (3) reproduces to Eq. (5).)

702 Thus, $b_{d i}$ is determined by the average spike history of the postsynaptic neurons over a 703 finite, presumably quite long, duration. Similarly, we can also generalize the evolution 704 equation for $q_{i j}$ as Eq. (4) in the main text. As demonstrated in Figure 2e and 2f, these 705 generalizations rarely decrease the learning accuracy of the algorithm.

707 The following is a possible biological implementation of our algorithm. (i) Each neuron 708 in the network continuously evaluates its membrane potential, $v$, and bias, $b$, and 709 stochastically generates spikes with the probability given in Eq. (1). (ii) A generated spike 710 is immediately integrated into the membrane potentials of its postsynaptic neurons, while 711 it slowly modulates the excitability of its presynaptic neurons (Eq. (2)). (iii) The spike 712 firing also modulates the latent synaptic variable, $q$ (Eq. (4)). (iv) The state of each 713 synapse is changed asynchronously and irregularly in accordance with Eq. (3) with a 714 frequency that is sufficiently slow that sensory neurons receive a large variety of external 715 inputs during the average interval of the updates. 


\section{Numerical simulations}

718 All numerical simulations are written in Python, with the open-source matrix library

719 CuPy. In details of the procedures to train a feedforward network are as follows. (i) We

720 first prepare $N D$ binary variables for the $N$ neurons and $W K$ synapses where $D$ is the

721 number of data in the training dataset, $W$ is the number of connections in the network,

722 and $K$ is the number of synapses per connection. (ii) Then we fix the variables of the

723 visible neurons to the values of the data in the training dataset, and initialize the values of

724 the hidden neurons and synapses randomly to 0 or 1 with probability $1 / 2$. (iii) To avoid

725 perfectly synchronized updates, we randomly choose the ratio $r_{x}$ for the hidden neurons

726 and update their variables according to Eqs. (1) and (2). (iv) Similarly, we randomly

727 choose the ratio $r_{s}$ for the $W K$ synapses to update in accordance with Eqs. (3) and (4).

728 (iv) We repeat (iii) and (iv) as many times as desired. The procedure to obtain the

729 prediction of the network is the same as that for the training procedure, except that we fix

730 only the input neurons and update the hidden and output neurons in accordance with Eqs.

731 (1) and (2), keeping the synaptic values fixed. In order to accelerate the computation, we

732 can use the average activities of the neurons, $\sigma\left(v_{d i}\right)$, instead of their binary variables,

$733 x_{d i}$, and omit the biases, $b_{d i}$, during the prediction procedure. The training and test

734 accuracies are defined as the ratio of the number of inputs that enables the network to

735 generate the correct outputs to the total numbers of inputs of the training and test datasets.

\section{Dataset}

738 Except in the cases described by Figs. 2 and 7, we used the MNIST dataset, which 739 consists of a training dataset of 60,000 examples and a test dataset of 10,000 examples in 740 which each image has $28 \times 28$ pixels. Because pixels in the MNIST data range from 0 to 741255 , we replaced them with 0 or 1 , depending on whether the value of the pixel is below 742 or above $255 / 2$. We thus obtain 784 -dimmensional binary input vectors.

744 In the situation considered in Fig. 2, we trained a three-layered network consisting of 40 745 input, 40 hidden, and 2 output neurons to learn a simple task that is a noisy and 746 high-dimensional variant of the XOR problem. The datasets were artificially generated as 747 follows. We first prepared two-dimensional binary vectors $\left(X_{d 1}, X_{d 2}\right)$, where $d$ is the 748 data index of the dataset. Then, to obtain 40-dimensional binary input vectors 
$749\left(Y_{d 1}, \cdots, Y_{d 40}\right)$, we set $Y_{d i}=X_{d 1}$ for $i=1, \cdots, 20$ and $Y_{d i}=X_{d 2}$ for $i=21, \cdots, 40$,

750 and then flipped their values randomly with a probability of 0.1 to obtain randomized

751 input dataset. The desired outputs of the two-dimensional vectors are given by $Z_{d i}=$

$752(0,1)$ if $\operatorname{XOR}\left(X_{d 1}, X_{d 2}\right)=0$ and $(0,1)$ if $\operatorname{XOR}\left(X_{d 1}, X_{d 2}\right)=1$. The training dataset and

753 test dataset each contains of 400 examples.

754

755 In the situation considered in Fig. 7, we used datasets consisting of temporal sequences to 756 train recurrent networks. Let us write the input data and desired outputs at time $\mathrm{t}$ as $757 X_{d i}(t)$ and $Z_{d i}(t)$. These are fed into the network by fixing the neurons in the input layer 758 as $x_{0, d i}(t)=X_{d i}(t), 0<i \leq N_{0}$, and those in the output layer as $x_{2, d i}(t)=Z_{d i}(t)$, $7590<i \leq N_{2}$, where $N_{0}$ and $N_{2}$ are numbers of neurons in the input and output layers, 760 respectively. A dataset is prepared as follows. We first prepare an integer sequence $s(t)$, 761 where $1 \leq s(t) \leq S$ and $1 \leq t \leq T$. We then set $X_{i}(t)=1$ if $(s(t)-1) N_{0} / S<i \leq$ $762 s(t) N_{0} / S$ and $X_{i}(t)=0$ otherwise. The target output is set as $Z_{d i}(t)=X_{i}(t+1)$ if $7631 \leq t<T$ and $Z_{d i}(T)=X_{i}(0)$ otherwise. To obtain randomized input vectors $X_{d i}(t)$, 764 we replicated $X_{i}(t)$ and randomly flipped them as $X_{d i}(t)=X_{i}(t)$ with probability 0.9 and $X_{d i}(t)=1-X_{i}(t)$ with probability 0.05 . The integer sequence $s(t)$ given by $1,2,3,4,5,4,3,2,1,2,3,4,5,4,3,2$ with $N_{0}=250, S=5$ and $T=16$ was used as the first dataset and $1,1,2,2,3,3,2,2,1,1,2,2,3,3,2,2,1,1,2,2,3,3,2,2$ with $N_{0}=150, S=3$ and $T=24$ is used for the second dataset.

\section{Parameters}

We used $r_{q}=1.0, r_{s}=0.001, K=200, a_{0}=0.1$, and $D=100$ in the situation considered in Fig. 1e, and $r_{b}=r_{q}=r_{x}=1.0, r_{s}=0.001, m_{0}=m_{1}=10, K=50$, $a_{0}=0.5$, and $D=400$ in the situation considered in Fig. 2 . In the situation considered in the remaining figures, except Figs. 4, 5, 6 and 7, we used $r_{b}=0.01, r_{x}=0.9$, $r_{q}=0.1, r_{s}=0.001, m_{0}=m_{1}=20, K=100$, and $a_{0}=0.1$. In the case of Fig. 4, we use $K=200$, and in the case of Figs. 5 and 6 , we used $r_{x}=0.5$, and in the case of Fig. 7, we used $r_{b}=0.1, r_{x}=1, r_{q}=0.01, r_{s}=0.01$ and $D=4000$. The numbers of hidden neurons in the three-layered network that are not specified in the figures are 1000 for Figs. 3f and 4, 100 for Figs. 5 and 6, and 500 for Fig. 7. Connection probability was 1.0 and 0.5 for feed forward connections and recurrent connections, respectively. 


\section{Data analysis}

\section{Spectrum variance of principle components}

784 After we trained the three-layered neural network considered in Fig. 3a using the MNIST

785 dataset, we fixed the synapses and obtained the average activities of the hidden neurons

$786\left\{\sigma\left(v_{d i}\right)\right\}_{i \in H}$. Note that the quantities $v_{d i}$ for the hidden neurons were deterministic in

787 this case because both the input neurons and the synaptic connections from them were

788 fixed. The principle component analysis was applied to the average activities after they

789 were standardized. Then we obtained the explained variance of each principle component,

790 which is the eigenvalue of the covariance matrix of the standardized average activities,

791 and ordered them in descending order. The exponent of the power law was estimated with

792 a least-square linear fit of the variance spectrum in log-log space.

\section{Statistics of network motifs}

795 We trained a three-layered recurrent network with 100 hidden neurons. Then, we 796 determined the number of connection patterns among the triplets of neurons over all 797 possible combinations of 3 neurons chosen from 100 , i.e. for $100 \cdot 99 \cdot 98 / 6=$ 798161,700 triples. Here, we only counted connections whose synaptic weights were 799 greater than or equal to 0.27 , in order to exclude small and negative connections. Null

800 hypothesis of the counts is defined as the same way that provided in the paper [31].

801 Namely, we determined the numbers of unidirectional and bidirectional connections in all

802 pairs of neurons and calculated the predicted number of three-neuron patterns by

803 assuming all constituent pairs of neurons in each triplet pattern are chosen independently,

804 while maintaining the probabilities of the measured unidirectional and bidirectional

805 connections. We performed 20 learning trials in order to obtain the mean and standard

806 deviation, $\sigma$, of the ratio of the actual number of each triplet pattern to that obtained with

807 the null hypothesis.

809 Limit of large training data size

810 Let us represent all free variables of a network consisting of neurons $\left\{x_{d i}\right\}$ and synapses $811\left\{s_{i j k}\right\}$ collectively by $\theta$. We can reasonably assume that the prior distribution $P(\theta)$ is 812 positive for all values of $\theta$ and that each training datum is independently generated from

813 a data distribution $P_{d}(x)$. Then, the posterior distribution satisfies 


$$
\begin{aligned}
P\left(\theta \mid\left\{x_{d}\right\}\right) & \propto \prod_{d} P\left(x_{d} \mid \theta\right) P(\theta) \\
& =\exp \left(\sum_{d} \log P\left(x_{d} \mid \theta\right)+\log P(\theta)\right) \\
& \cong \exp \left(N_{d} \int \log P(x \mid \theta) P_{d}(x) d x+\log P(\theta)\right)
\end{aligned}
$$

814 which generally converges to a delta function $\delta\left(\theta-\theta_{0}\right)$ in the limit of a large number of 815 training data, $N_{d} \rightarrow \infty$, where $\theta_{0}=\operatorname{argmax}_{\theta} \int \log P(x \mid \theta) P_{d}(x) d x$. (If maximum is 816 realized of multiple values of $\theta$ simultaneously, the posterior distribution will converge 817 to the sum of the corresponding delta functions.) 
bioRxiv preprint doi: https://doi.org/10.1101/811646; this version posted January 19, 2020. The copyright holder for this preprint (which was not certified by peer review) is the author/funder, who has granted bioRxiv a license to display the preprint in perpetuity. It is made available under aCC-BY-NC-ND 4.0 International license.

a

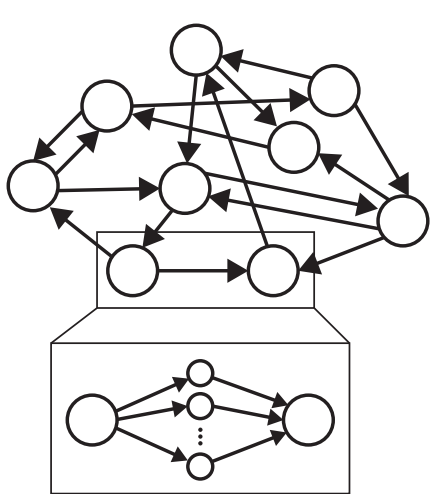

b

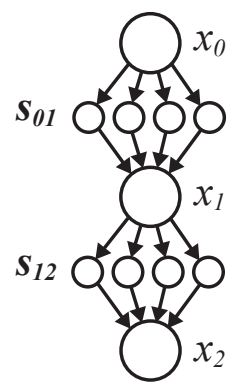

C

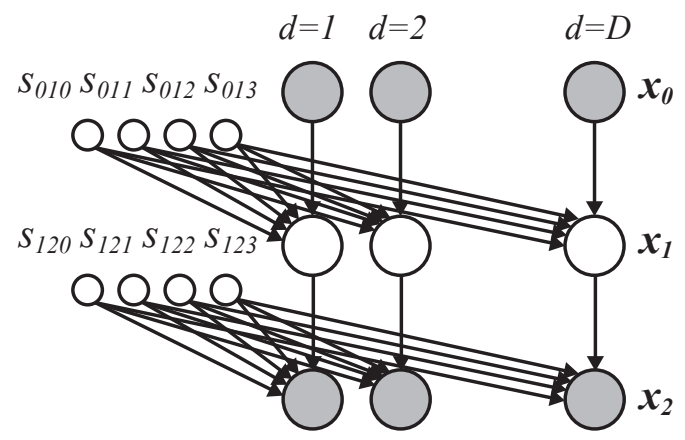

d

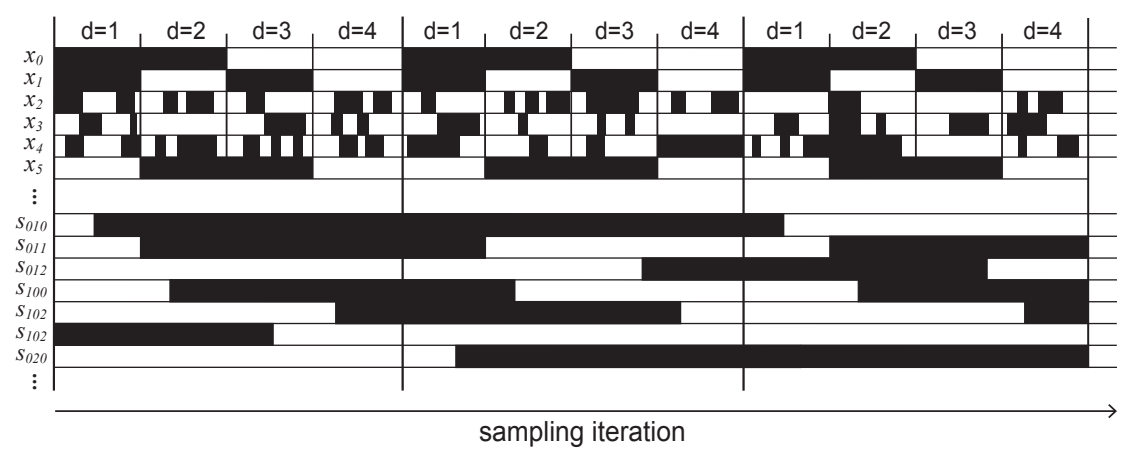

e
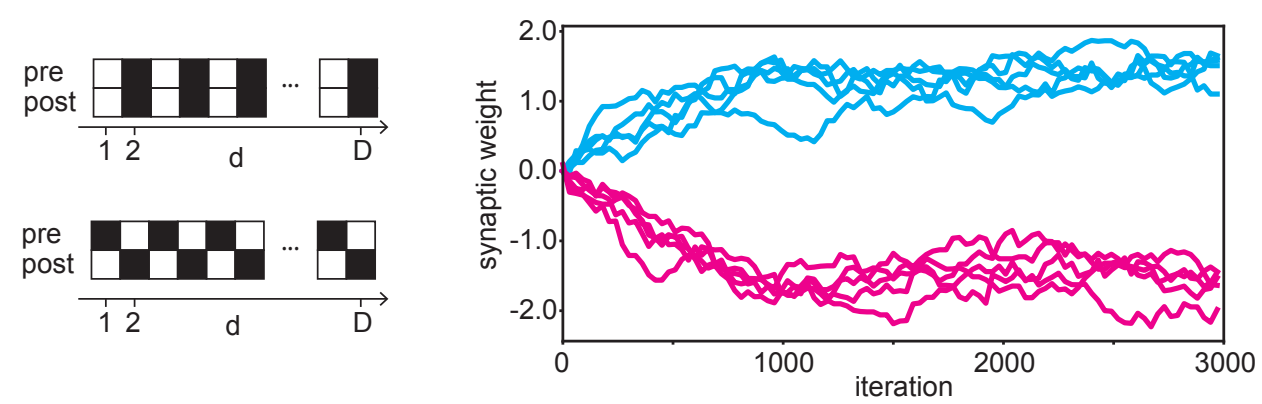
bioRxiv preprint doi: https://doi.org/10.1101/811646; this version posted January 19, 2020. The copyright holder for this preprint (which was not certified by peer review) is the author/funder, who has granted bioRxiv a license to display the preprint in perpetuity. It is made available under aCC-BY-NC-ND 4.0 International license.

a

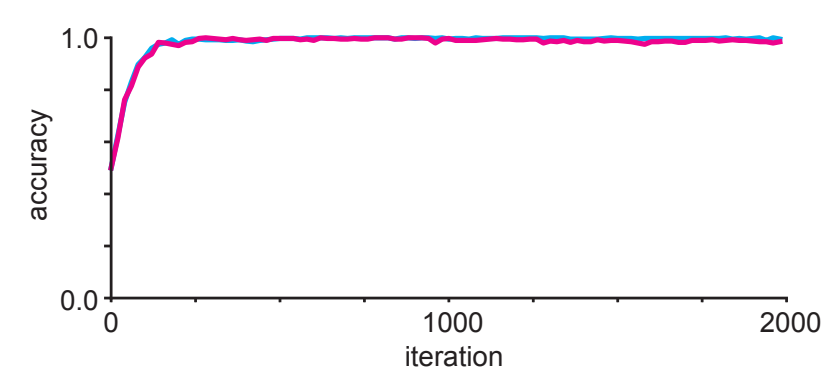

b

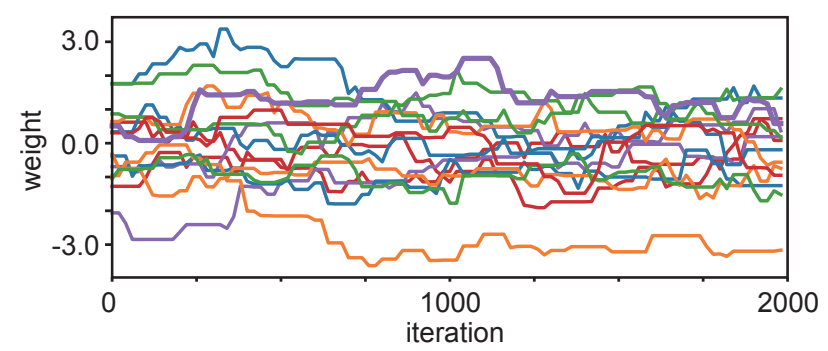

C

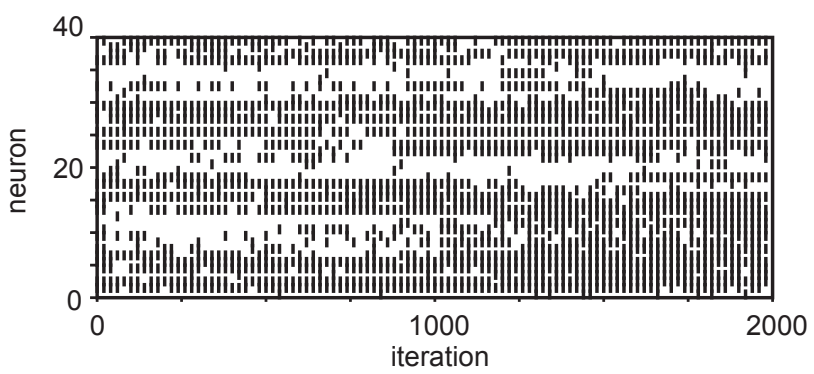

d

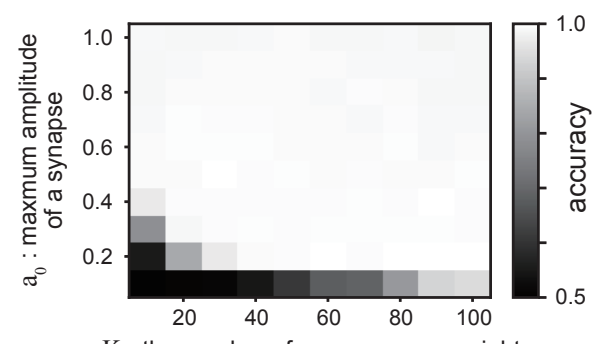

$\mathrm{K}$ : the number of synapses per weight
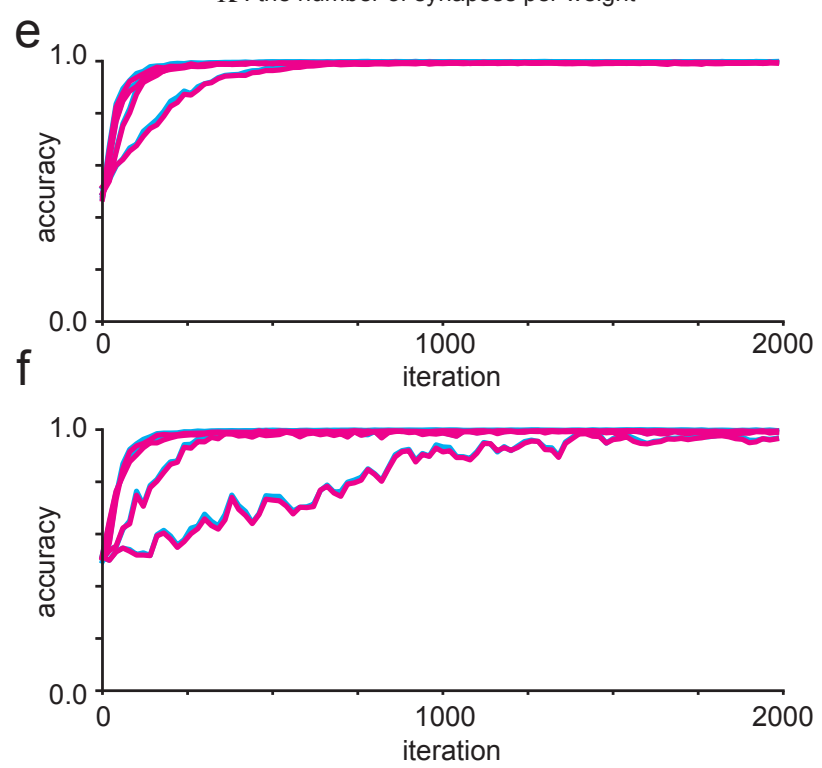
bioRxiv preprint doi: https://doi.org/10.1101/811646; this version posted January 19, 2020. The copyright holder for this preprint (which was not certified by peer review) is the author/funder, who has granted bioRxiv a license to display the preprint in perpetuity. It is made available under aCC-BY-NC-ND 4.0 International license.

a

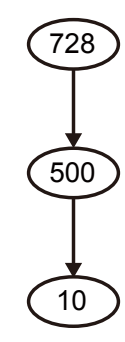

b

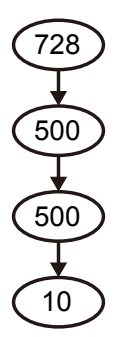

c
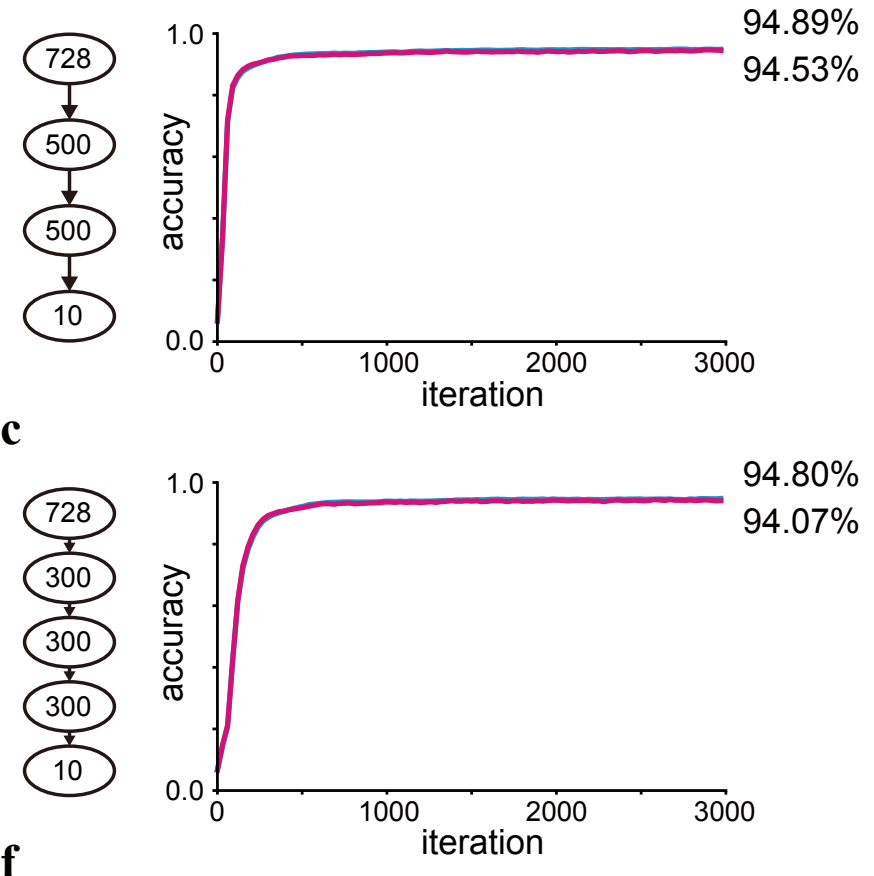

f
$96.07 \%$

95.56\%

d

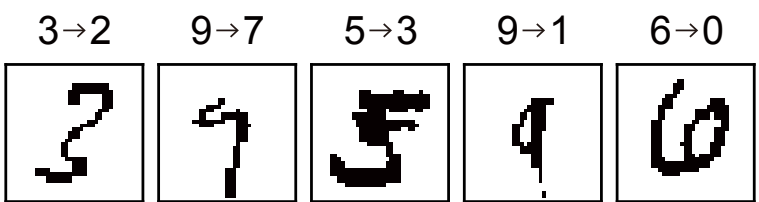

$4 \rightarrow 8$

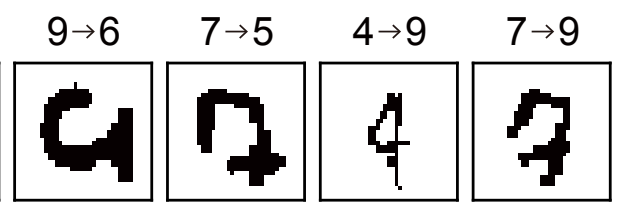

e
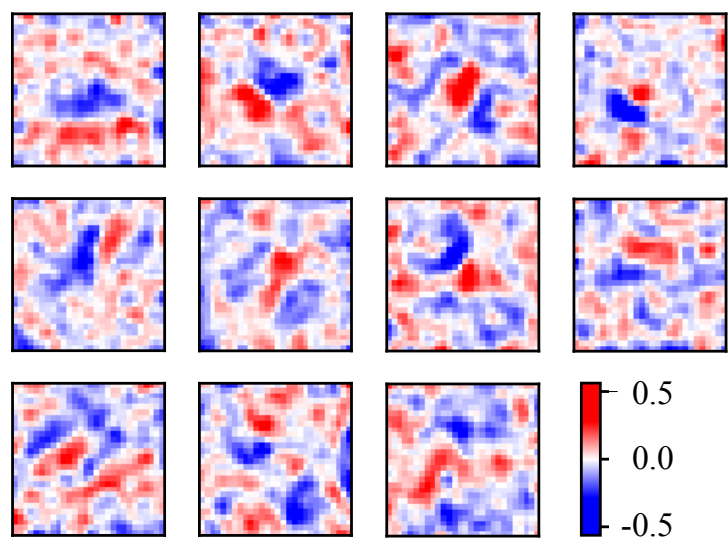

0.5

0.0

$-0.5$
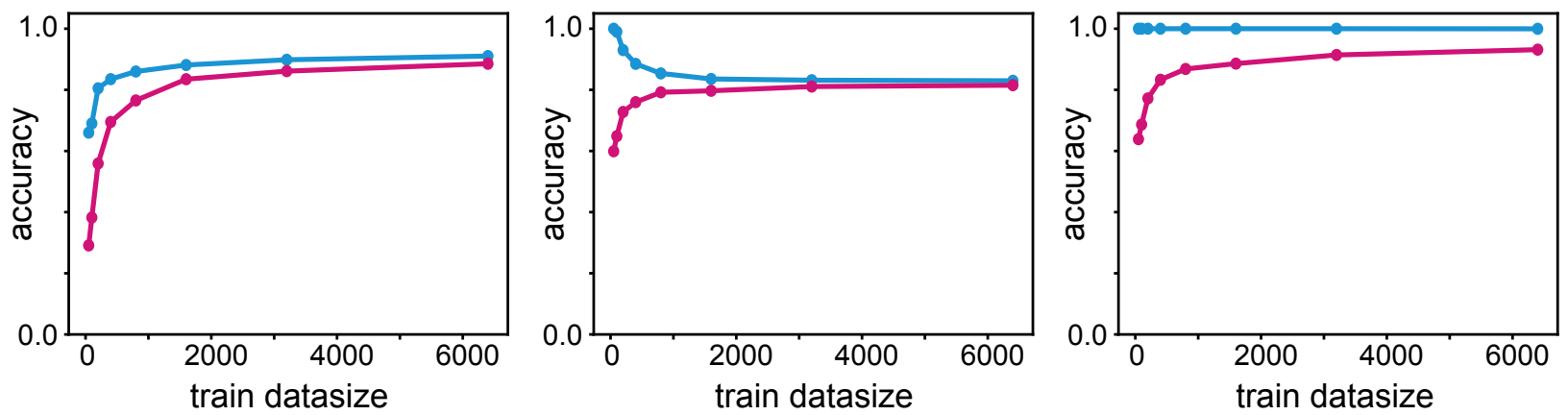
bioRxiv preprint doi: https://d do مorg/101101/811646; this version posted January 19,2020. The copyright holder for this preprint (which was not certified by peer reie th)is the author/funder, who has granted bioRxiv alligen se to display the preprint in perpetuity.ttis made available under
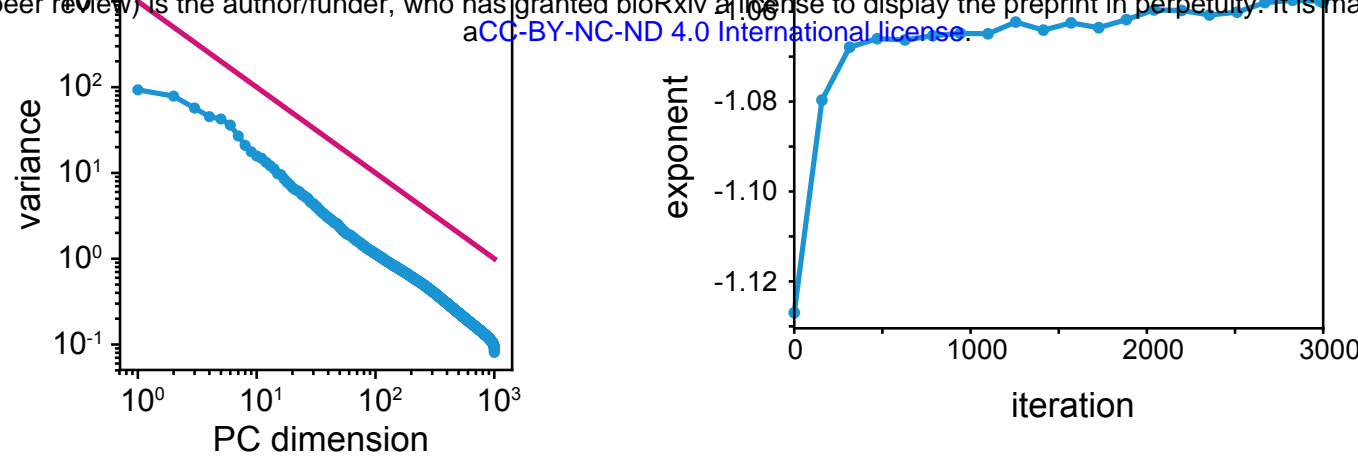
bioRxiv preprint doi: https://doi.org/10.1101/811646; this version posted January 19, 2020. The copyright holder for this preprint (which was not certified by peer review) is the author/funder, who has granted bioRxiv a license to display the preprint in perpetuity. It is made available under aCC-BY-NC-ND 4.0 International license.

a

b

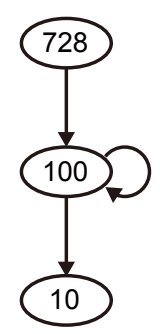

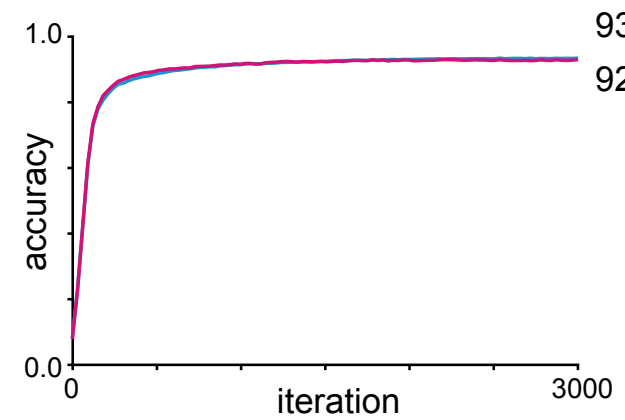

C

$93.32 \%$

$92.28 \%$

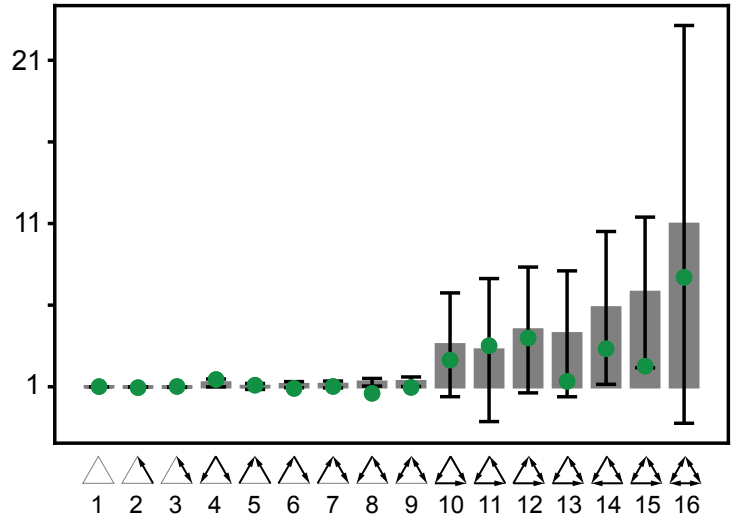


bioRxiv preprint doi: https://doi.org/10.1101/811646; this version posted January 19, 2020. The copyright holder for this preprint (which was not certified by peer review) is the author/funder, who has granted bioRxiv a license to display the preprint in perpetuity. It is made available under aCC-BY-NC-ND 4.0 International license.

a

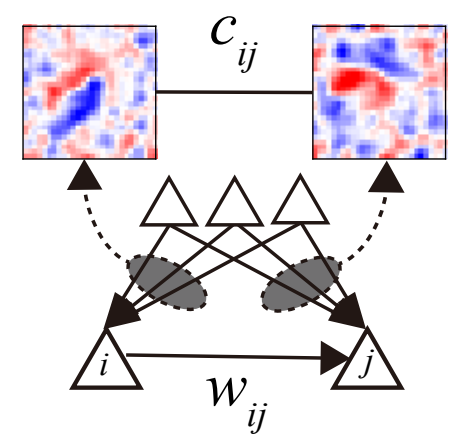

b

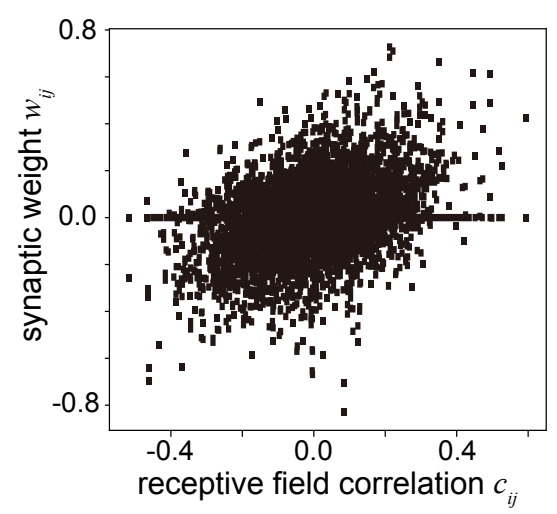

C

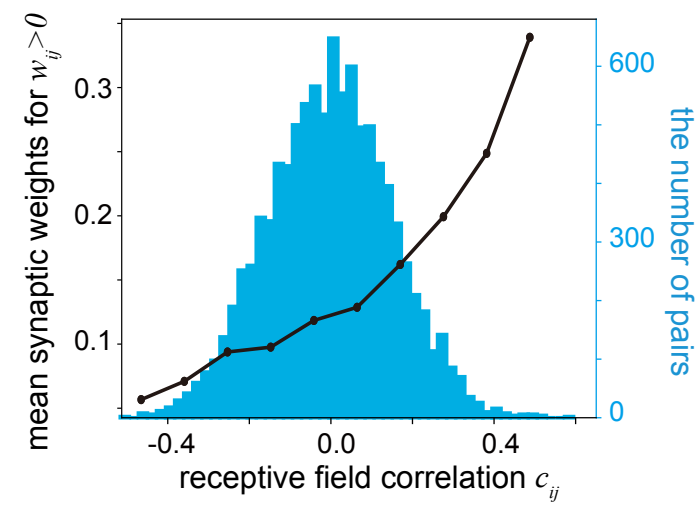


bioRxiv preprint doi: https://doi.org/10.1101/811646; this version posted January 19, 2020. The copyright holder for this preprint (which was not certified by peer review) is the author/funder, who has granted bioRxiv a license to display the preprint in perpetuity. It is made available under aCC-BY-NC-ND 4.0 International license.

a

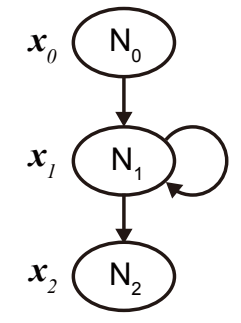

C

iteration $=0 \quad$ data

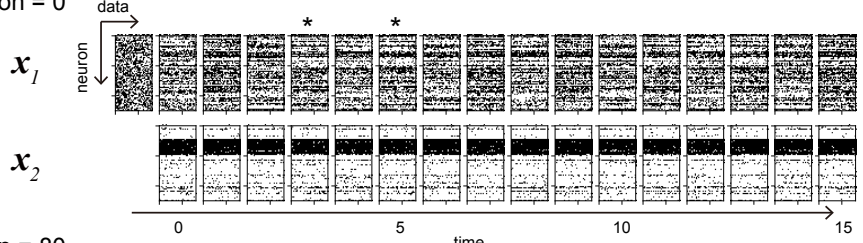

iteration $=80$

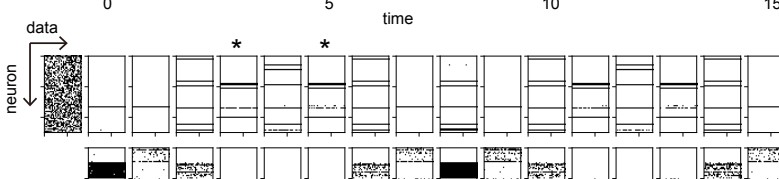

$\boldsymbol{x}_{2}$

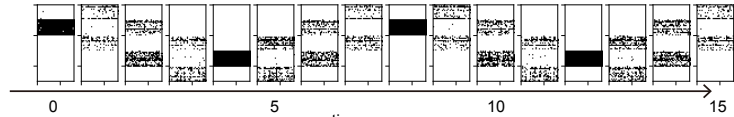

iteration $=400$

time

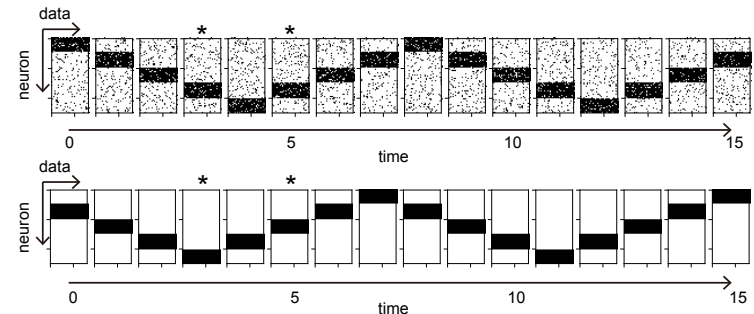

e

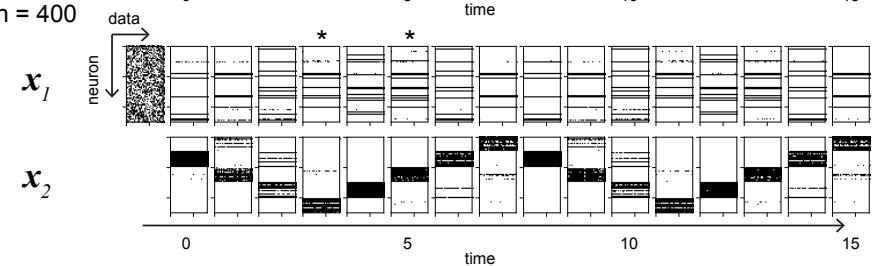

d

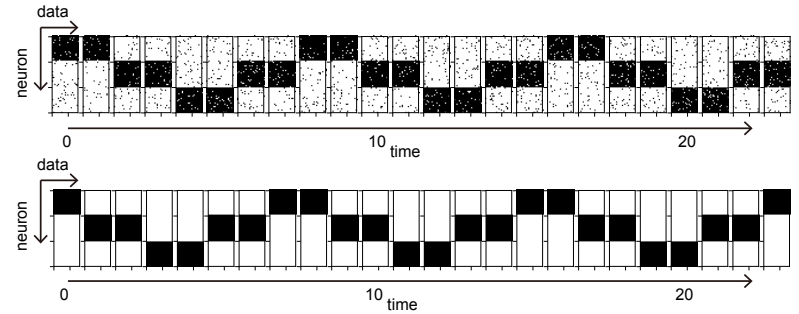

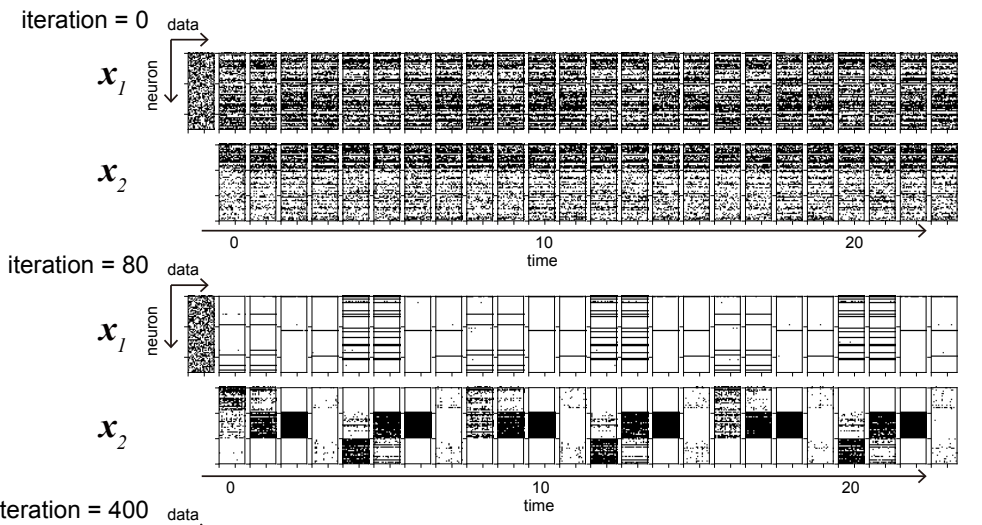

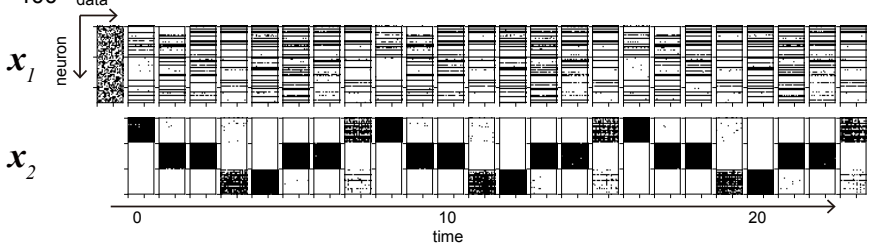

